\title{
Negotiating Varied Research Goals in Computing Education Research
}

\author{
Mark Guzdial \\ Electrical Engineering \& CS Dept. \\ University of Michigan \\ Ann Arbor, MI, USA \\ mjguz@umich.edu
}

\author{
Colleen M. Lewis \\ Computer Science Dept. \\ Harvey Mudd College \\ Claremont, CA, USA \\ lewis@cs.hmc.edu
}

\author{
Lauren Margulieux \\ Dept. of Learning Sciences \\ Georgia State University \\ Atlanta, GA, USA \\ lmargulieux@gsu.edu
}

\author{
Greg L. Nelson \\ Computer Science \& Engineering Dept. \\ University of Washington, Seattle \\ Seattle, WA, USA \\ glnelson@uw.edu
}

\author{
Leo Porter \\ Computer Science and Engineering Dept. \\ University of California, San Diego \\ La Jolla, CA, USA \\ leporter@eng.ucsd.edu
}

\begin{abstract}
As we celebrate the 50th SIGCSE Symposium, this panel explores how computing education researchers chart a course individually and as a community to build our research practices and collective knowledge of computing education. This navigation involves developing our research goals, which tools we use to work towards those goals, and which academic communities outside of computing education we seek to learn from and contribute to. However, these processes of navigation are rarely discussed as a community. Paper and grant submissions and reviews provide an imperfect way for our community to communicate our varied values and priorities. This panel brings together experts in computing education research who differ in their research goals, tools, and external communities. We can expect a lively discussion amongst the panelist and we hope to spark important discussions within the computing education research community!
\end{abstract}

\section{KEYWORDS}

Research goals, research practices and methods, interdisciplinary

\section{PANEL STRUCTURE}

In honor of the 50th SIGCSE Symposium, this panel considers the various fields that contribute to computing education research (CER) and how researchers integrate diverse goals, tools, and products in their research. This panel brings together experts in CER who differ in their academic training, professional goals, and memberships in external communities.

Permission to make digital or hard copies of part or all of this work for personal or classroom use is granted without fee provided that copies are not made or distributed for profit or commercial advantage and that copies bear this notice and the full citation on the first page. Copyrights for third-party components of this work must be honored. For all other uses, contact the owner/author(s).

SIGCSE '19, February 27-March 2, 2019, Minneapolis, MN, USA

(c) 2019 Copyright is held by the owner/author(s)

ACM ISBN 978-1-4503-5890-3/19/02.

https://doi.org/10.1145/3287324.3287329
15 minutes: The panelists will provide background about the goals, tools, and products that define their research.

20 minutes: The panelists will discuss strengths, weaknesses and limitations of their approach, and collaborations that they have with researchers who take different approaches.

30 minutes: The panel will invite attendees to contribute questions of individual panelists or for the panelists to discuss.

10 minutes: The panel will close with brief comments regarding how they would like CER to evolve over the next 10 to 30 years.

\section{GOALS, TOOLS, AND PRODUCTS}

CER, like other discipline-based educational research, brings together people with different goals:

- practitioners (i.e., educators and curriculum and tool developers) who primarily want to optimize learning,

- discipline-based education researchers who primarily want to understand and improve learning within computing, and

- general education researchers who primarily want to understand how people learn in general.

Each group values the goals of the other groups. In addition, researchers can belong to multiple groups or collaborate across groups, but they must negotiate their priorities to determine the research tools that they should use.

- action research is a rapid method to implement and evaluate design decisions and improve learning environments but it does not normally include many scientific controls, making it difficult to explain why changes occur or generalize findings.

- design-based research (DBR) tests designs of learning environments, tools, or interventions that are based on theory. In the early stages of DBR, the focus is on improving design with a specific context, and in the later stages, the focus shifts to building generalizable theory.

- highly-controlled experiments examine factors that affect learning independently from other contextual factors to 
isolate the effects of variables in the learning environment and build general learning theory.

The tools that are used throughout a project can greatly impact the products of the work and how external communities engage it.

- action research generally produces directly applicable solutions, such as tools and instructional materials for a specific context (i.e., course, topic, or school) and can provide insight for education researchers,

- early stage DBR generally produces domain-specific theory and design of learning environments, tools, and interventions of interest to discipline-based ed researchers and can guide practice and development or application of learning theory,

- late stage DBR and highly-controlled experiments produce global theories or interventions that generalize across topics and domains and guide practice and future research.

Each of these research goals, tools, and connections to external communities provide valuable contributions to CER. We will explore the benefits and challenges of different approaches. We will also explore how to contribute to and communicate in research projects that include multiple approaches.

\section{POSITION STATEMENTS}

\subsection{Mark Guzdial, University of Michigan}

Position: I am a computer scientist who did a PhD in CS and Education. I was trained as a learning scientist who worked in computing. I recognize that I need to collaborate with educational psychologists and other learning scientists who know research methods and theories that I don't. I can provide them with insights into computing, a field with growing importance for all learners.

Expertise and background: Mark Guzdial is a professor in CS \& Engineering and Engineering Education Research. His focus is on making computing available to a wide audience, particularly nonCS majors, end-user programmers, and high school teachers. He tends to publish in ACM SIGCSE venues, but he sometimes publishes in the education and learning sciences communities.

\subsection{Colleen Lewis, Harvey Mudd College}

Position: I will focus on my experience as a practitioner and my work documenting and disseminating knowledge about teaching CS (i.e., pedagogical content knowledge or PCK) through CSTeachingTips.org. As an educator, general and domain-specific theory inform my actions, but may never provide the granularity to guide every decision. While examples of CS PCK are often dismissed as anecdotes, these anecdotes can match the granularity of my decisions and help me notice and respond to important aspects of my students' learning.

Expertise and background: Colleen Lewis is the McGregorGirand Associate Professor of CS. Trained as a learning scientist and teaching CS, her scholarship is spread across these boundaries and builds upon research in social psychology and higher ed.

\subsection{Lauren Margulieux, Georgia State Univ.}

Position: As a learning scientist, my primary research goals are to build general theory for how people develop cognitive skills and develop interventions to improve learning outcomes. I often work with discipline-based education researchers to conduct research in authentic learning environments. This approach allows my research to make both applied contributions within a domain and theoretical contributions to the learning sciences. I have found that satisfying the needs of both general and domainspecific research goals takes extra design and development time but leads to high quality and well-devised research and products. Expertise and background: Lauren Margulieux is an assistant professor in the Department of Learning Sciences. Her training is in cognitive psychology, instructional design, and learning technologies. She conducts research on problem solving in STEM. In addition to ACM SIGCSE, she is a member of the International Society of the Learning Sciences and American Educational Research Association.

\subsection{Greg Nelson, University of Washington}

Position: As a pragmatic design-focused researcher, I prioritize finding more effective designs for learning computing. I have written about tensions between pursuing design and explanatory research goals. I create and evaluate learning tools, including discontinuous designs like learning to read a programming language first instead of learning reading and writing at the same time. I also develop domain-specific theories of what computing knowledge is and assessments for them, such as program tracing. Expertise and Background: Greg Nelson is a senior CS $\mathrm{PhD}$ student under Andy Ko, trained in HCI, computing education, and statistics. His research focuses on assessment and instructional design for computing education, including tutorial systems and other learning environments beyond in-person classrooms.

\subsection{Leo Porter, UC San Diego}

Position: I am a computer scientist whose $\mathrm{PhD}$ is in computer architecture. Having moved into CS education in 2011, I bring the focus on benchmarking, standards, and empirical research from architecture to my research in CS education. On the panel, I will focus on my work evaluating the effectiveness of Peer Instruction in CS and in ongoing work predicting which students are at-risk and which factors lead to them being at-risk. I have found that empirical research based on data from measurable outcomes and from qualitative studies with students both helps us better understand the impact of known phenomena while discovering new lines of inquiry.

Expertise and background: Leo Porter is an Associate Teaching Professor. He teaches a wide variety of classes across the curriculum including courses on introductory programming, upper division data structures and architecture, and graduate courses on architecture and CEd research. His research focuses on evaluating pedagogical practices, developing instruments to measure student learning, and predicting and understanding atrisk students. 